\title{
Research Paper \\ Effect of COVID-19-induced Home Quarantine on Parental Stress and its Relation- ship With Anxiety and Depression Among Children in Guilan Province
}

\author{
Sajjad Rezaei ${ }^{1}$ (i), Azadeh Sameni Toosarvandani ${ }^{1}$ (i), ${ }^{*}$ Azra Zebardast $^{1}$ ()ㅜ
}

1. Department of Psychology, Faculty of Literature and Humanities, University of Guilan, Rasht, Iran.

\begin{tabular}{|c|c|}
\hline $\begin{array}{l}\text { Use your device to scan } \\
\text { and read the article online }\end{array}$ & dtation Rezaei S, Sameni Toosarvandani A, Zebardast A. Effect of COVID-19-induced Home Quarantine on Parental Stress \\
\hline 口清实回 & and its Relationship with Anxiety and Depression Among Children in Guilan Province. Iranian Journal of Psychiatry and Clinical \\
\hline Fist & Psychology. 2020; 26(3):280-293. http://dx.doi.org/10.32598/ijpcp.26.3402.1 \\
\hline Iftis & 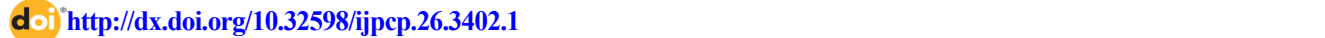 \\
\hline
\end{tabular}

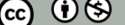

Received: 02 May 2020 Accepted: 13 Jul 2020 Available Online: 01 Dec 2020

Key words:

Quarantine, Anxiety, Depression, COVID-19, Child, Life change events

\begin{abstract}
A B S T R A C T
Objectives Acute attacks of epidemics and the physical risk have adverse severe psychological effects on children due to their lower protective capacity.

This study aimed to determine home quarantine's psychological effect because of Coronavirus (COVID-19) on parental stress and its relationship with anxiety and depression in children.

Methods This research was conducted during 17-26 March 2017 - the initial stages of the Iranian people's public call for quarantine - in Guilan province. The study's statistical population included all children aged 5 to 12 years and their parents who were purposefully sampled, and 181 people responded voluntarily to the Child Symptom Inventory-4 (CSI-4)-Parental Form and the Impact of Event Scale-Revised (IES-R). For data analysis, the Pearson correlation coefficient, independent $t$-test, and regression analysis were used.

Results After eliminating the effect of demographic variables, it was found that more parents) scores on the intrusion subscale $(\beta=0.568, P=0.004)$ and hyperarousal $(\beta=0.772, P<0.0001)$ could predict more anxiety scores. None of the IES-R components in parents could predict children's depression scores $(P>0.05)$. In general, the higher parental scores on IES-R, the more likely the child to have anxiety scores $(\beta=0.258$, $P=0.011)$ and depression $(\beta=0.325, P<0.0001)$ in children.

Conclusion Based on the results, it can be said that the psychological effect of home quarantine caused by Covid-19 pandemic in parents can have a devastating impact on children's anxiety and depression, and these results necessitate the training programs of psychological support for parents and their children.
\end{abstract}

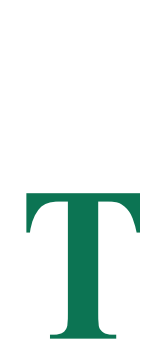

\section{Extended Abstract}

\section{Introduction}

he outbreak of Coronavirus Disease 2019 (COVID-19) turned this disease into the world's largest health threat in 2019. According to the World Health Organization (WHO), COVID-19 is a respiratory infectious disease caused by the newest known coronavirus, unrecognized before the initiation of the outbreak in $\mathrm{Wu}-$ han, China, in December 2019. Given coronavirus's lifethreatening nature, most training and services are focused on medical aspects, albeit with a reasonable justification. However, based on psychoneuroimmunology related theories, experience, and tolerance, maladaptive to stress can weaken the immune system and lead to an increased risk of disease development. It should also be noted that the virus survivors face serious psychological threats during and after the disease eradication.

ㄷ.

${ }^{*}$ Corresponding Author:

Azra Zebardast, PhD.

Address: Department of psychology, Faculty of Literature and Humanities, University of Guilan, Rasht, Iran.

Tel: +98 (911) 1558479

E-mail: zebardast@guilan.ac.ir 
Most of the psychological studies available on this emerging disease are related to the adult population, such as students, the general public, and health care staff in hospitals involved with coronavirus patients. In contrast, a very small number of studies have been performed on children. Given the importance of psychological attention to children in the current psychological and health crisis, the present study intended to investigate the psychological effect of COVID19-induced home quarantine on parental stress and its relationship with children's anxiety and depression Guilan Province, Northern Iran.

\section{Method}

This research was conducted 7-16 March 2020, in the early stages of the public call to observe the quarantine in Guilan Province, Iran. The statistical population was all children in Guilan Province aged 5-12 years and their parents. The children were enrolled through the purposive sampling method. A total of 180 quarantined parents participated in the study. The inclusion criteria were living in Guilan Province, having a child aged 5-12 years, and completing an online questionnaire. The exclusion criterion was the history of anxiety and depression in the child diagnosed by a psychologist or a psychiatrist. The data were collected through a researcher-made demographic questionnaire, the items related to anxiety and depression on the Children Symptom Inventory (CSI-4), and the Weiss and Marmer Impact of Event ScaleRevised (IES-R). The data collection tools were shared on social media (Telegram and WhatsApp). The collected data were analyzed through the Pearson correlation coefficient, independent t-test, and hierarchical regression analyses after adjusting demographic confounders' effect.

\section{Results}

This online survey's findings showed that $96.7 \%$ and $3.3 \%$ of the parents who completed the questionnaires $(n=181)$ were mothers and fathers with a mean age of 36.48 years and 40.43 years, respectively. The mean age of children whose parents participated in the study was 6.99 years. The analysis was performed on 5-12 years old children, $51.9 \%$ of whom were girls and $48.1 \%$ were boys. After eliminating the effect of demographic variables (number of children in family and age of the child), it was revealed that high scores of parents on subscales intrusion $(\beta=0.568$, $\mathrm{P}=0.004)$ and hyperarousal $(\beta=0.772, \mathrm{P}<0.0001)$ can significantly predict high scores of children's anxiety. Besides, none of the IES-R components in parents could substantially predict children's depression scores $(\mathrm{P}>0.05)$. After eliminating the effect of variables(number of children in the child's family and age), higher IES-R scores of parents significantly predicted higher anxiety scores of children $(\beta=0.258, P=0.011)$. Finally, the results showed that higher IES-R scores could substantially predict children's higher depression scores $(\beta=0.325, \mathrm{P}<0.0001)$ (Table $1 \& 2)$.

\section{Discussion and Conclusion}

It seems that Fear of illness, home quarantine-induced financial problems, temporary or permanent unemployment, limited social connections, unknown end of the pandemic, concern for family and relatives, and Fear of the future are among the factors that, as COVID-19 pandemic-induced adverse psychological effects, may cause stress in parents. This stress can predict anxiety and depression in children. Home quarantine increases interactions between parents and children, although this increase in the quantity can be associated with a decline in communication quality.

Table 1. Hierarchical multivariate linear regressions to predict scores of anxiety and depression based on IES-R sub-scales

\begin{tabular}{|c|c|c|c|c|c|c|c|}
\hline Criteria & Steps and Variables & $\mathbf{R}^{2}$ & $R^{2}$ Change & F Change & Beta to Enter ${ }^{a}$ & $\mathbf{P}$ & $\mathrm{DW}^{\mathrm{b}}$ \\
\hline \multirow{4}{*}{$\begin{array}{l}\vec{d} \\
\frac{\vec{x}}{\vec{x}}\end{array}$} & $\begin{array}{l}\text { 1. Demographics } \\
\text { Number of children }\end{array}$ & 0.059 & 0.047 & 4.757 & 0.243 & 0.032 & \\
\hline & 2. IES-R subscales & & & & 0.149 & 0.155 & 1.901 \\
\hline & Avoidance Intrusion & 0.278 & 0.219 & 7.384 & 0.568 & 0.004 & \\
\hline & Hyperarousal & & & & 0.772 & 0.0001 & \\
\hline \multirow{4}{*}{ 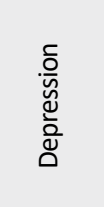 } & $\begin{array}{l}\text { 1. Demographics } \\
\text { Age of the child }\end{array}$ & 0.022 & 0.022 & 3.966 & 0.147 & 0.048 & \\
\hline & 2. IES-R subscales & & & & 0.096 & 0.206 & 1.906 \\
\hline & Avoidance Intrusion & 0.177 & 0.155 & 11.078 & 0.232 & 0.069 & \\
\hline & Hyperarousal & & & & 0.207 & 0.122 & \\
\hline
\end{tabular}

${ }^{\mathrm{a}}$ Standardized regression coefficient; ${ }^{\mathrm{b}}$ Durbin-Watson test. 
Table 2. Hierarchical multivariate linear regressions to predict scores of anxiety and depression based on IES-R total score

\begin{tabular}{|c|c|c|c|c|c|c|c|}
\hline Criterion & Step and Variable & $\mathbf{R}^{2}$ & $R^{2}$ Change & F Change & Beta to Enter a & $\mathbf{P}$ & $\mathrm{DW}^{\mathrm{b}}$ \\
\hline \multirow[t]{2}{*}{ Anxiety } & $\begin{array}{l}\text { 1. Demographics } \\
\text { Number of children }\end{array}$ & 0.059 & 0.047 & 4.757 & 0.243 & 0.032 & \multirow[t]{2}{*}{1.901} \\
\hline & 2. IES-R total score & 0.138 & 0.079 & 6.868 & 0.258 & 0.011 & \\
\hline \multirow{2}{*}{ Depression } & $\begin{array}{l}\text { 1. DemographicsAge of the } \\
\text { child }\end{array}$ & 0.022 & 0.022 & 3.966 & 0.147 & 0.048 & \multirow{2}{*}{1.944} \\
\hline & 2. IES-R total score & 0.127 & 0.105 & 21.492 & 0.325 & 0.0001 & \\
\hline
\end{tabular}

${ }^{a}$ Standardized regression coefficient; ${ }^{\mathrm{b}}$ Durbin-Watson test.

Parents who experience stress caused by quarantine or the disease outbreak are more likely to have individual and responsible concerns about their children or the withheld mandatory supports for their parents or others. They are also faced with hyperarousal and disturbing thoughts, which hinder a good quality relationship with children and possibly cause significant signs of anxiety and depression. This problem requires further investigations through quasiexperimental projects.

Given the importance of the issue, specific strategies have been developed by the countries' health agencies to provide psychological assistance to children. For example, the Iranian Academy of Child and Adolescent Psychiatry has provided guidelines on issues such as management of stress and excitement in crisis (stress and crisis management in the family, talking to the child about coronavirus disease, etc.), resuming academic activities and virtual education, psychological support for the sufferers (psychological support for the loss of loved ones, helping children mourn, the affected child: what parents should do to support themselves and their families, etc.), and long-term measures in crisis(timely recognition of risk signs and prevention, visiting specialists and counseling in cyberspace: tips for parents, etc.).

The results of this study confirmed the effects of unwanted thoughts and hyperarousal of parents on children; accordingly, psychotherapists should intervene with families to separately manage and treat stress and anxiety of parents and depression of children and to modify their possible maladaptive interactive styles through interactive and aggressive treatments, all of which can help mental and financial saving for the government and health care providers.

\section{Ethical Considerations}

Compliance with ethical guidelines

All ethical principles are considered in this article. The participants were informed of the purpose of the research and its implementation stages. They were also assured about the confidentiality of their information and were free to leave the study whenever they wished, and if desired, the research results would be available to them.

\section{Funding}

This research did not receive any specific grant from funding agencies in the public, commercial, or not-for-profit sectors.

\section{Authors' contributions}

Conceptualization and methodology: Sajjad Rezaei; Investigation and critical review: Azra Zebardast; Data collection and data analysis: Azadeh Sameni Toosarvandani

\section{Conflicts of interest}

The authors declare no conflict of interest. 


\title{
تأثير قرنطينه خانكى ناشى از ياندمى بيمارى كوويد 9 أبر استرس والدين و رابطه آن با اضطراب و افسردتى كودكان تيلانى
}

\author{
سجاد رضايى' هـ، آزاده ثامنى توسروندانى' هـ، "عذرا زبردست' (ه) \\ ا. كروه روانشناسى، دانشكده ادبيات وعلوم انسانى، دانشكاه كيلان، رشت، ايران.
}

\begin{abstract}
حكيد

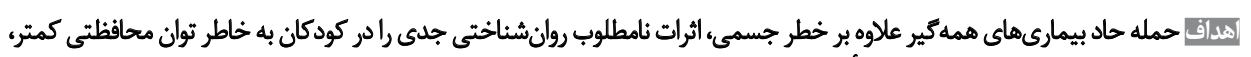

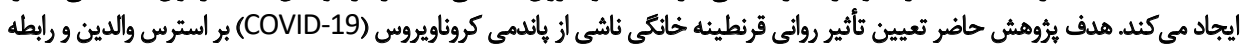

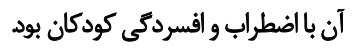

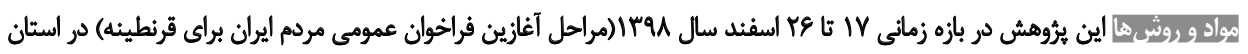

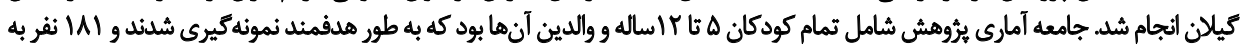

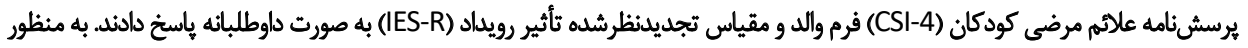

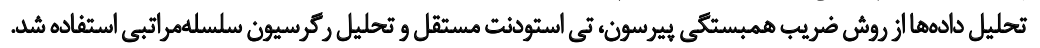

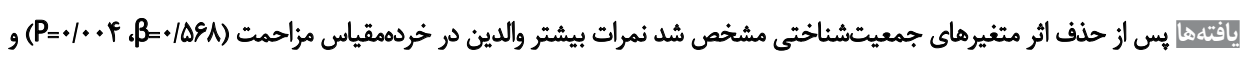

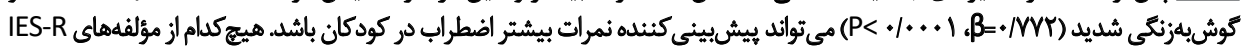

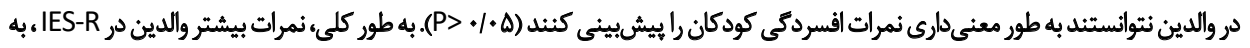

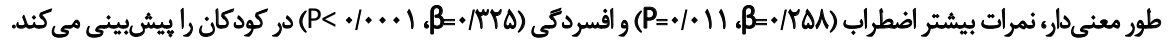

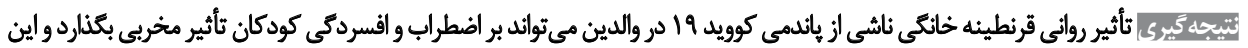

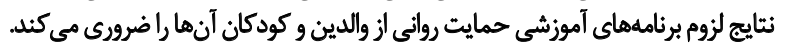

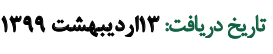

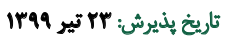

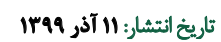

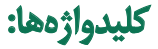

قرنطينه، اضطراب،

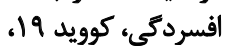
كودى، رويدادهاى تغيير زند5ى
مراكز آموزشى اعمم از مهد كودكىها، يبيشدبستانىها و مدارس

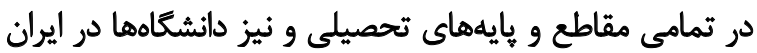

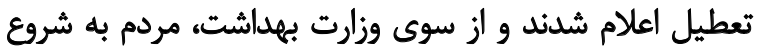

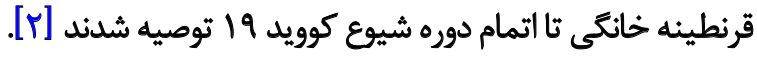

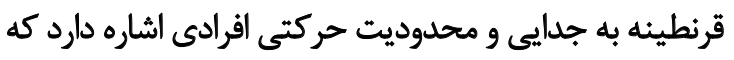

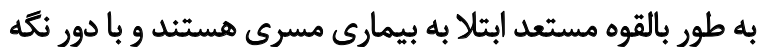

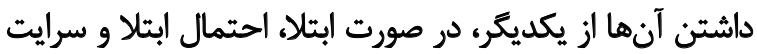

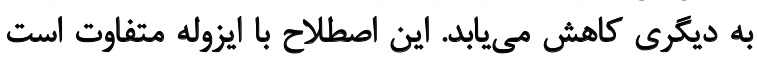

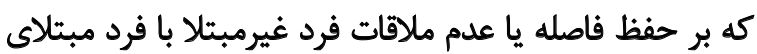

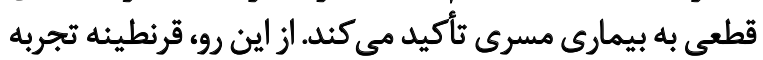

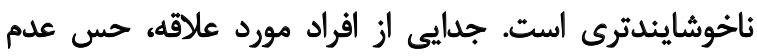

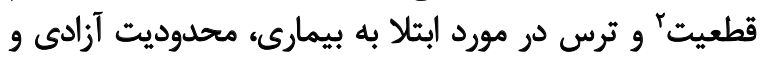

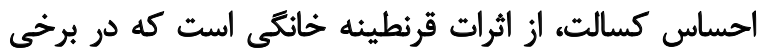

\section{Feeling insecure}

dales بيمارى كوويد 19 با شيوع كسترده در سال 19 •ب در سراسر

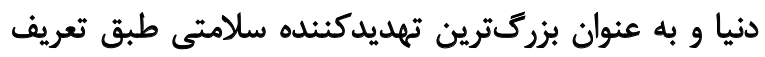

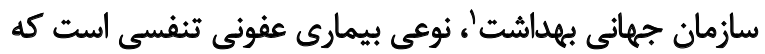

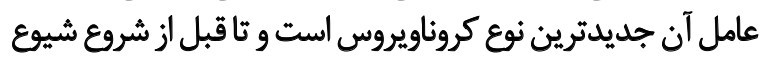

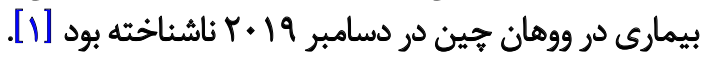
در ايرانء ابتدا نتيجه آزمايش دو مورد مشكوك به به كوويد 19 ادر

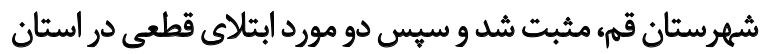

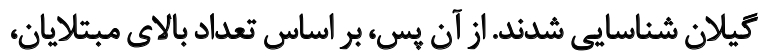

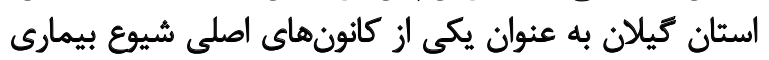

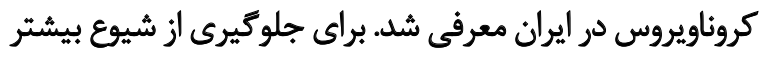

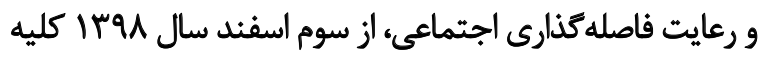

1. World Health Organization (WHO)

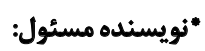

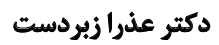

نشانىى: رشت، دانشكاه كيلان، دانشكده ادبيات و علوم انسانى، كروه روانشناسى. تلفن: بست الكترونيكى: zebardast@guilan.ac.ir 


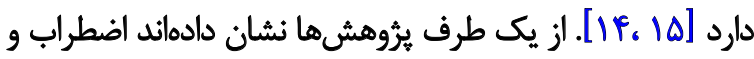

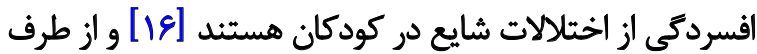

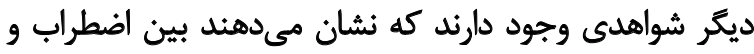

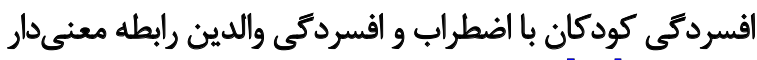

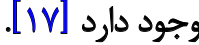

كروناويروس يك بيماري همه

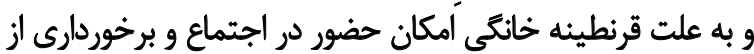

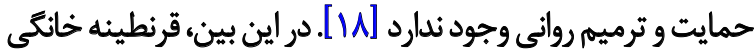

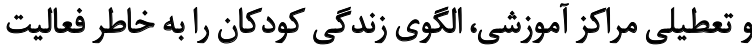

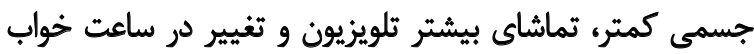

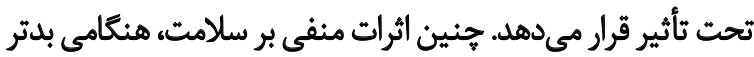

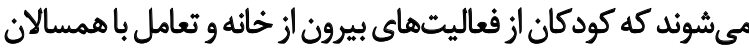

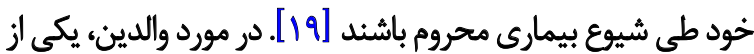

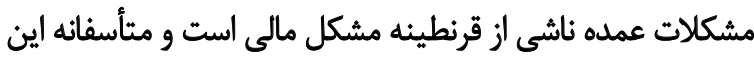

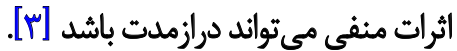
براى كودكانى كه در خانه با والدين يا نزديكان خود قرنطينه

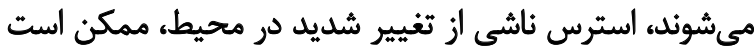

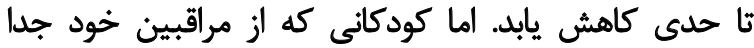

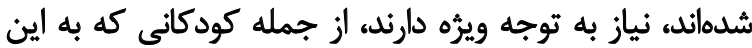

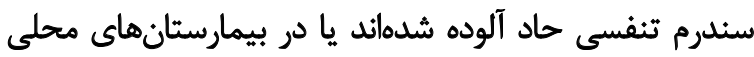

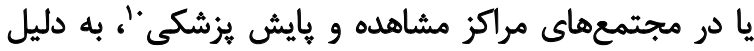

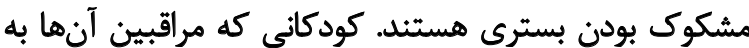

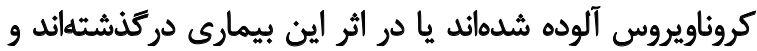

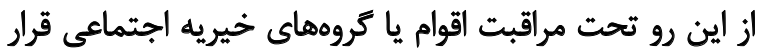

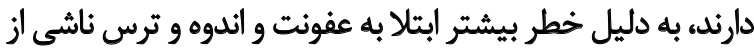

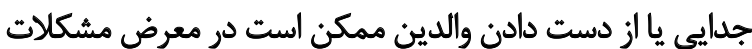

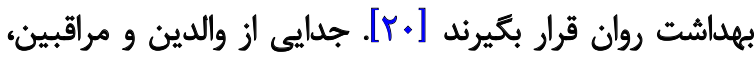

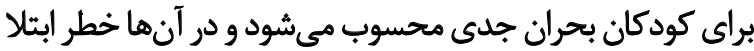

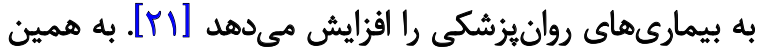

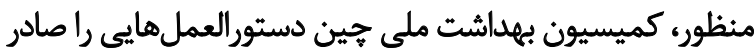

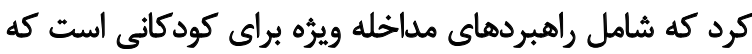

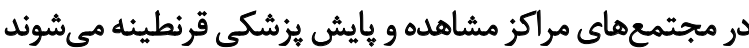

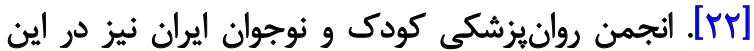

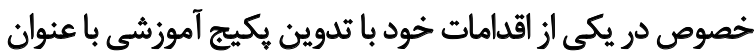

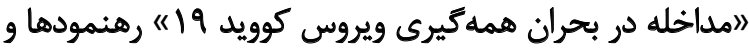

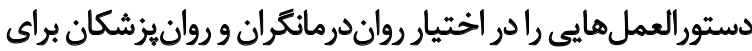

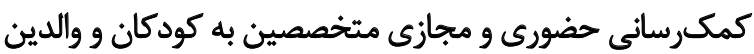

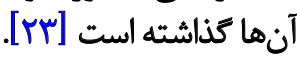

بر اساس نتايج ثيروهشهاى محدود و موجود انجامشده روى

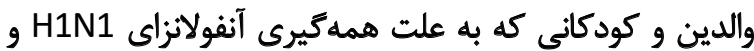

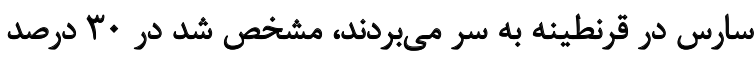

10. Collective Medical Observation Centers

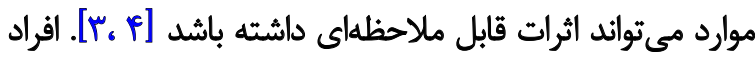

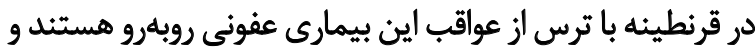

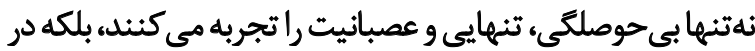

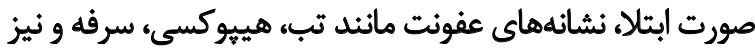

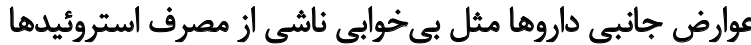

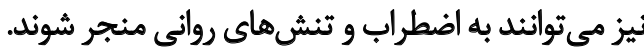

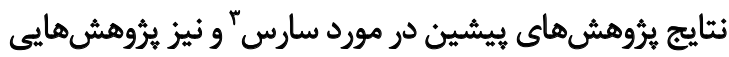

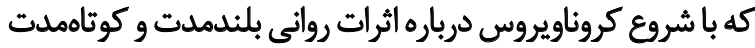

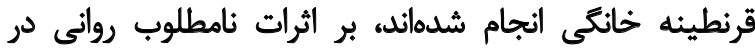

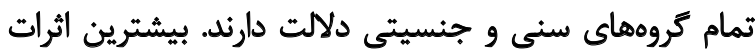

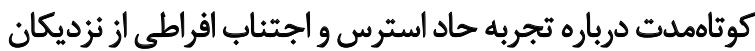

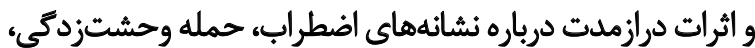

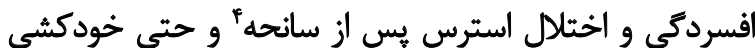

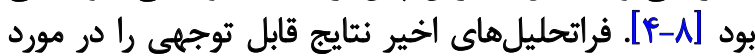

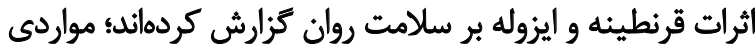

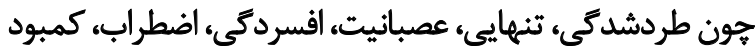

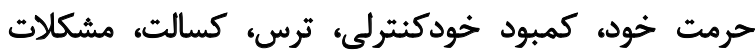
عاطفى، اختلال در امور روزمره و وتأثيرات منفى دردئ در كنار آمدن

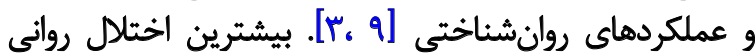

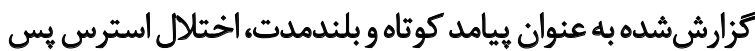

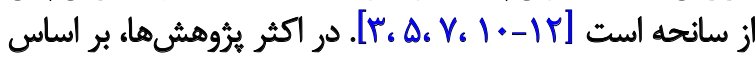

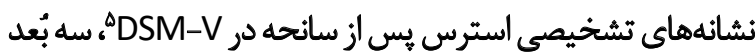

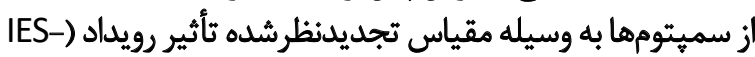

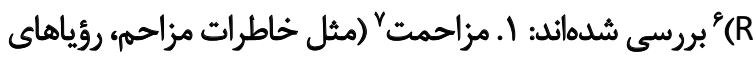

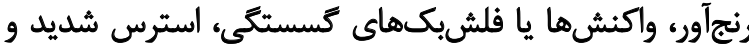

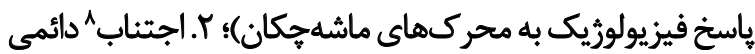

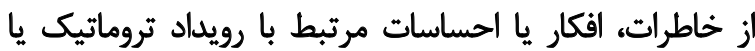

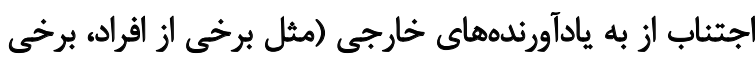

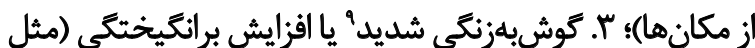

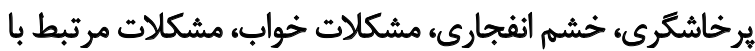
تمركز حواس، رفتار بى احتياط يا رفتار خودمخرب) همسو با DSM-V كه اختلالات اضطراب و افسردكى را با باليا

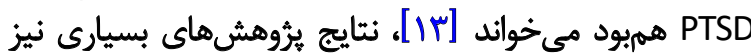

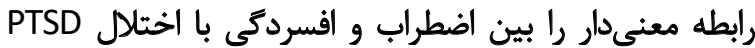

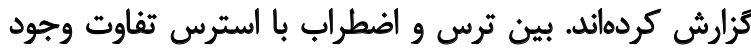

3. SARS

4. Post-Traumatic Stress Disorder (PTSD)

5. Diagnostic and Statistical Manual of Mental Disorder, $5^{\text {th }}$ Edition

6. Impact of Event Scale-Revised (IES-R)

7. Intrusion

8. Avoidance

9. Hyperarousal 
اسفند يعنى در مراحل آغازين فراخوان عمومى مردم ايران براى

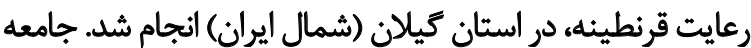

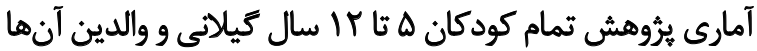

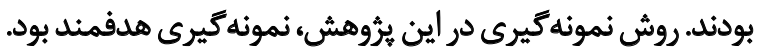

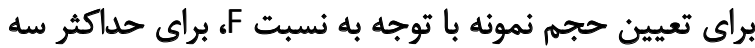

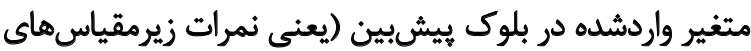
و و جهار متغير هميراش (شامل سن والدين، سن كودئ (IES-R

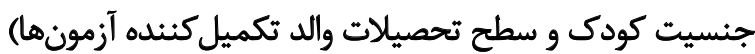

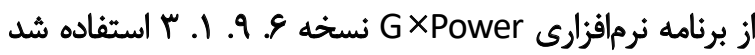

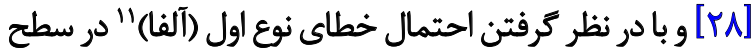

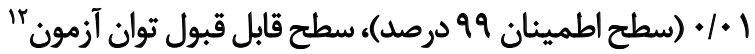

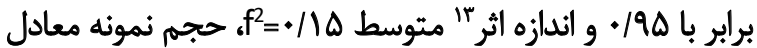

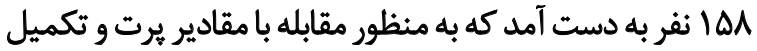

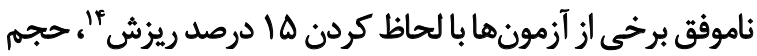

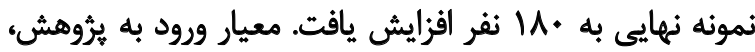

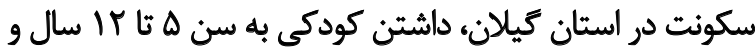

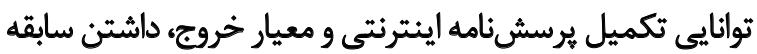

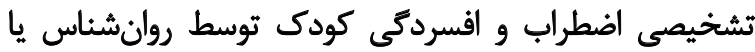

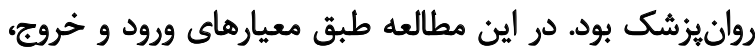

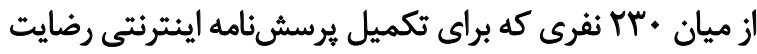

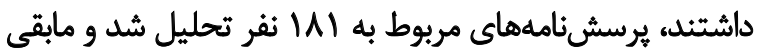

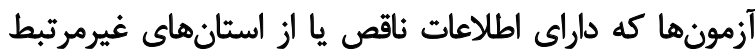
بودند، از تحليل آمارى خارج شدند.

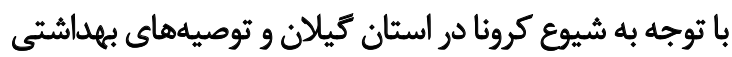

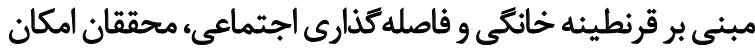

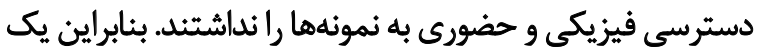

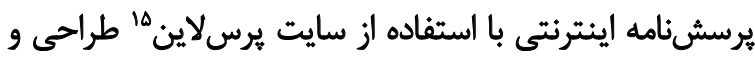

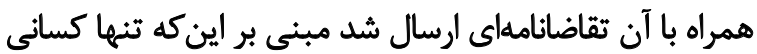

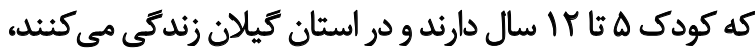

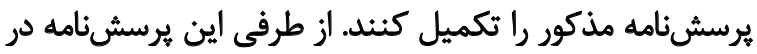

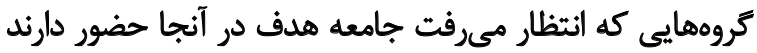

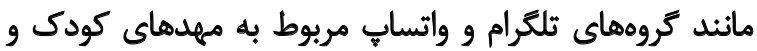

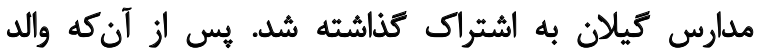

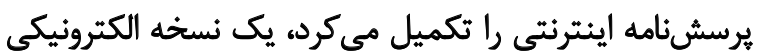

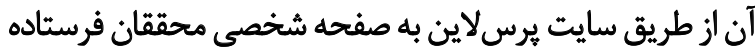
مىشد.

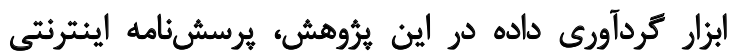

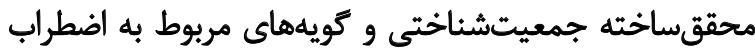

11. Type I Error (alpha)

12. Power

13. Effect size

14. Dropout

15. Available at: https://porsline.ir/

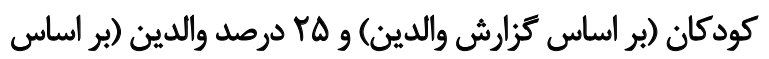

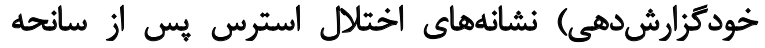
مشاهده شده است [1/].] اضطراب دوران كودكى به عنوان يكى از عوامل مؤثر در رشد

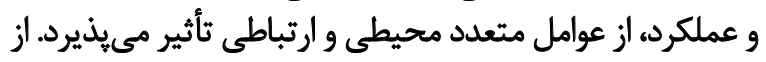

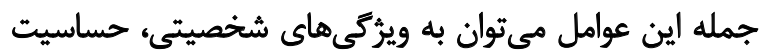

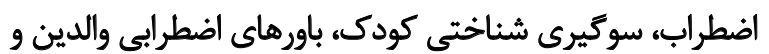

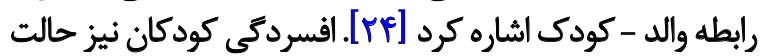

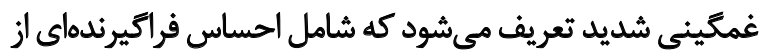

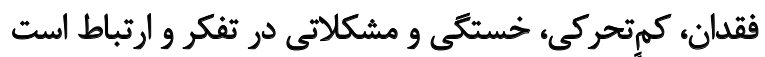

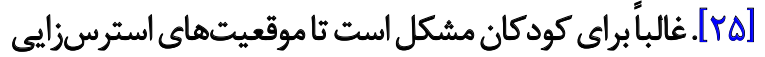

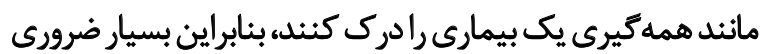

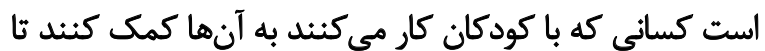

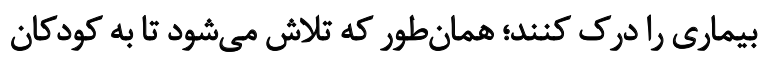

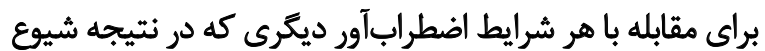

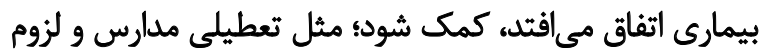

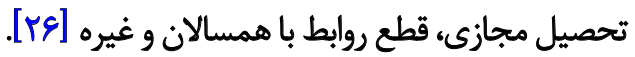

با توجه به تهديد جدى كرونا ويروس، بيشتر آموزشها و ورئ

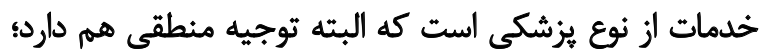

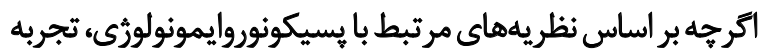

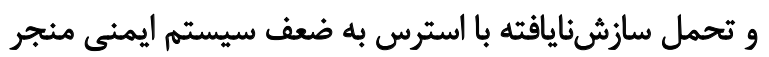

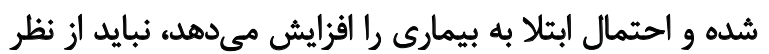

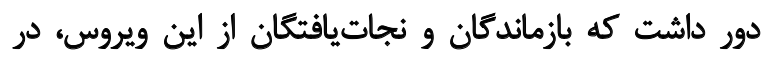

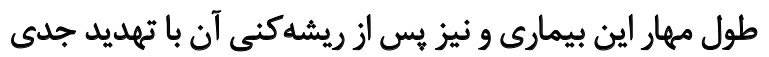

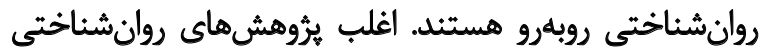

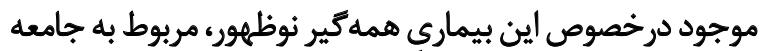

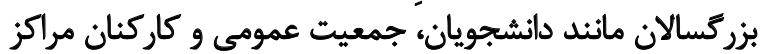

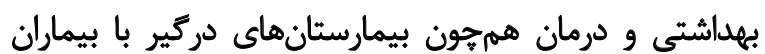

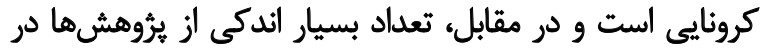

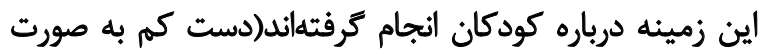

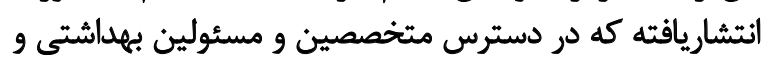

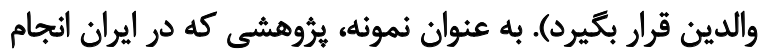

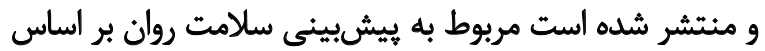

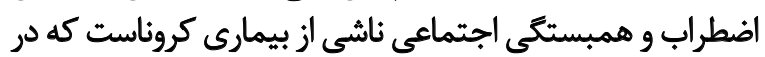

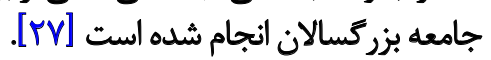
نظر به اهميت توجه روانشناختى به كودكان در شرايط بحران بان باني

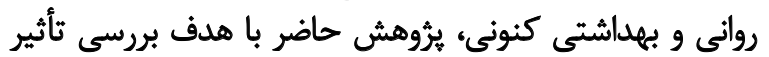

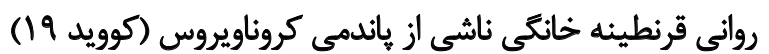

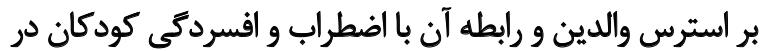

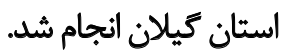

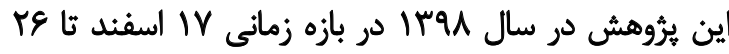


جدول ا. مشخصات جمعيتششناختى خانوادهها و كودكان مورد يُوهش

\begin{tabular}{|c|c|c|}
\hline فراوانى (درصد) & & \\
\hline $\begin{array}{l}\text { IVA (QSN) } \\
\&(r / T)\end{array}$ & مادر & جنسيت والد \\
\hline $\begin{array}{l}\text { qf }(\Delta \backslash / A) \\
\text { AY (FNI) }\end{array}$ & هختر & جنسيت كودى \\
\hline $\begin{array}{l}1 \cdot V(\Delta V /) \\
\operatorname{eV}(V V / 0)\end{array}$ & تكفرزندى & \\
\hline$r(M /)$ & سه فرزند & تعداد فرزندان خانواده \\
\hline $1(\cdot / \Delta \Delta)$ & جهار فرزند & \\
\hline$f(T / Y)$ & كزارش نشده & \\
\hline
\end{tabular}

جدول r. ماتريس ضرايب همبستكى بين نمرات كل IES-R و زيرمقياسهاى آن در والدين با نمرات اضطراب و افسردى كودكان

\begin{tabular}{|c|c|c|c|c|c|c|c|c|}
\hline 8 & $\Delta$ & $f$ & $r$ & $r$ & 1 & ميانكين \|انحرافمعيار & متغيرها & رديف \\
\hline & & & & & - & $r \Delta / T| \pm| \Phi / T \mid$ & تأثير رويداد & 1 \\
\hline & & & & - & $.19 y^{*}$ & $1 \% / 9 \Delta \pm \Delta / A V$ & اجتاب & $r$ \\
\hline & & & - & $\cdot \pi \Lambda^{* *}$ & $\cdot / \mathrm{Ar}$ & 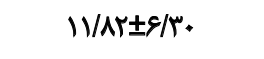 & مزاحمت & $r$ \\
\hline & & - & $\cdot / A p e r$ &.$/ 4 I^{* *}$ & ./9Y" & $\checkmark / \Delta r \pm s / 4 q$ & كوشبلهزنكى شديد & $r$ \\
\hline & - & ( ) &.$/ 1 e^{\circ}$ & . Kצ"* &.$/ T r$ & $1 / T V \pm \cdot / v \Delta$ & اضطراب & $\Delta$ \\
\hline- &.$/ \Delta q^{* *}$ &.$/ \Delta^{* * *}$ & 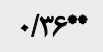 & $.1 .9^{*}$ &.$/ M * *$ & $\cdot / W \pm \cdot / T q$ & افسردكى & 8 \\
\hline
\end{tabular}

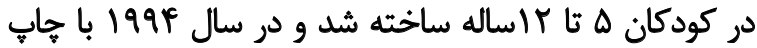

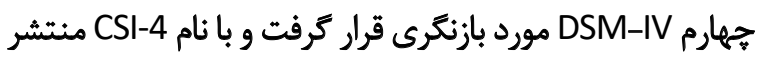

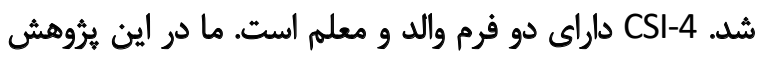

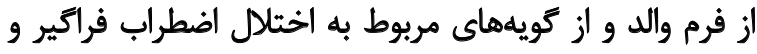

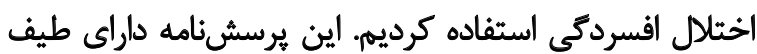

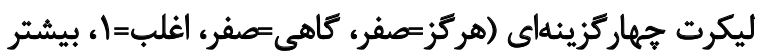

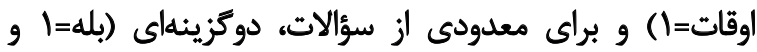

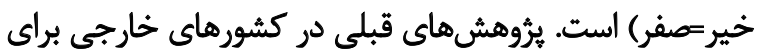

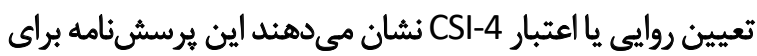

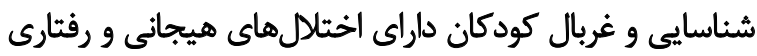

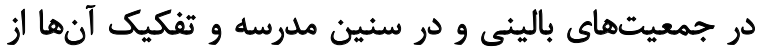

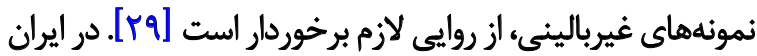

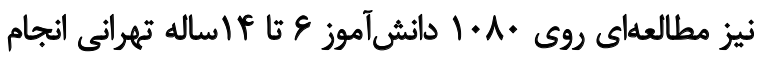

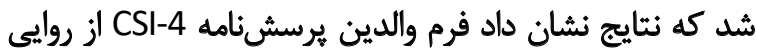

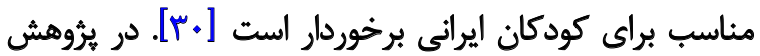

و وافسردگى يرسشنامه علائم مرضى كودكان" و و مقياس

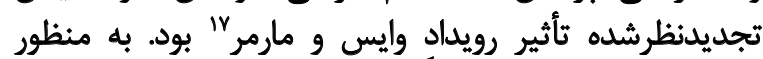

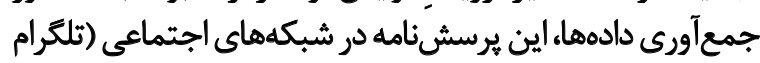

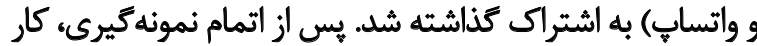
تجزيه و تحليل دادهها آغاز شد.

$$
\text { يرسش نامه علائم مرضى كودكان }
$$

اين يرسشنامه يك ابزار درجهبندى رفتار است كه براي

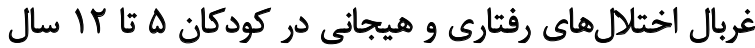

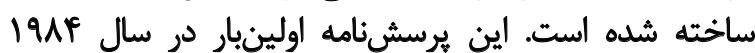

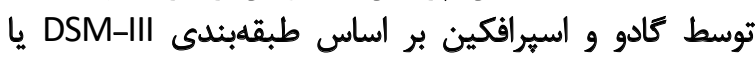

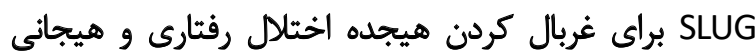

16. Children Symptom Inventory-4 (CSI-4)

17. Weiss \& Marmar (IES-R) 
جدول ب. ركرسيونهاى خطى سلسلهمراتبى براي يُشبينى نمرات اضطراب و افسردكى بر اساس ابعاد IES-R يس از كنترل عوامل مخدوش كننده جمعيتشناختى

\begin{tabular}{|c|c|c|c|c|c|c|c|}
\hline DW' & $\mathbf{P}$ & Beta to Enter & F Change & $\mathbf{R}^{2}$ Change & $\mathbf{R}^{2}$ & كامها و مثغيرها & متغير ملكى \\
\hline & $.1 \cdot M$ & . MTrY & F/VAV & . l. PV & $1 \% \Delta q$ & ا. جمعيتشناختي & \\
\hline
\end{tabular}

.$/ 9.1$

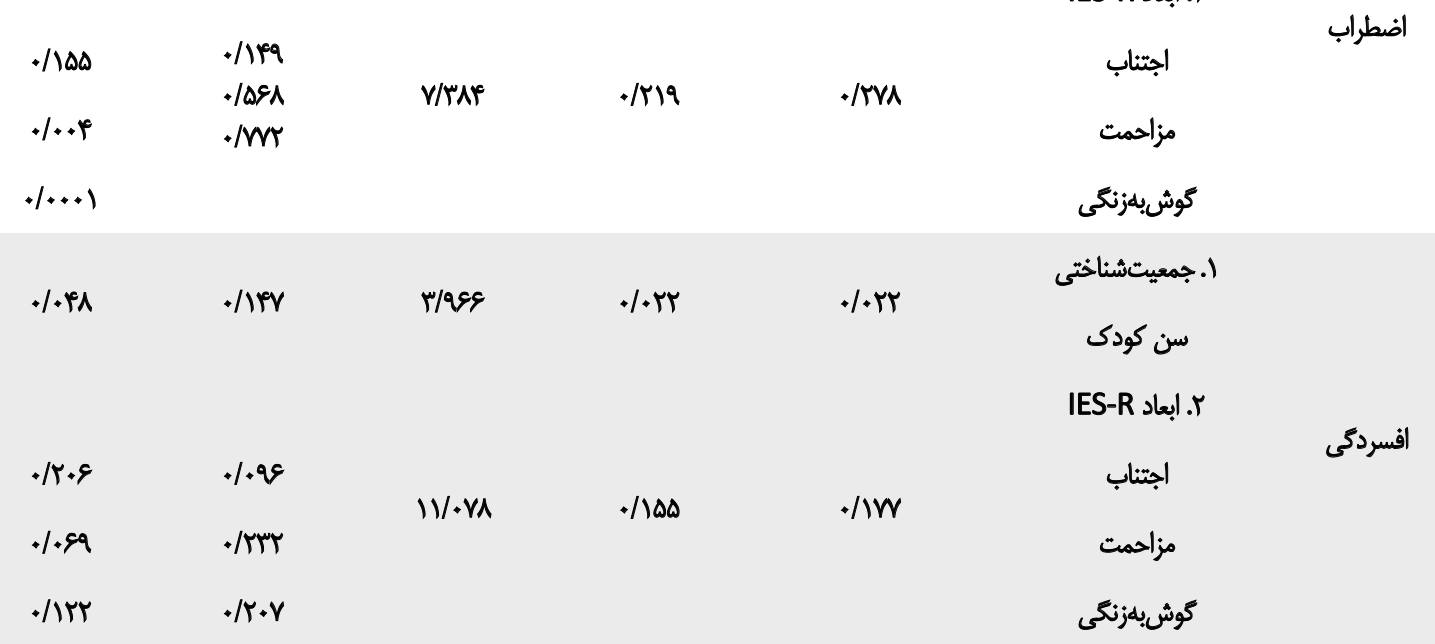

روايى ظاهرى يرسش نامه توسط يك روانيزشك و يك نفر فوق تخصص روانيزشكى كود كان مورد بررسى و تأييد قرار كرفت نئ.

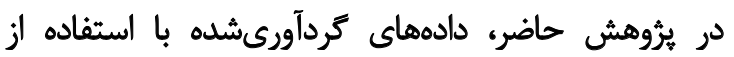

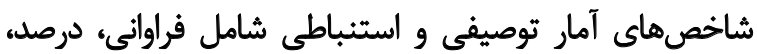

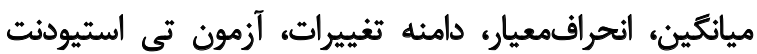

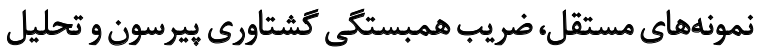

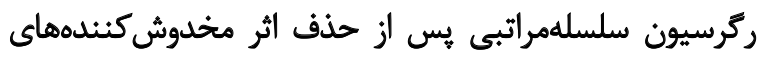

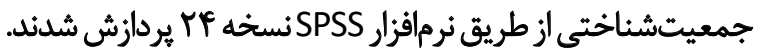

Ladiós

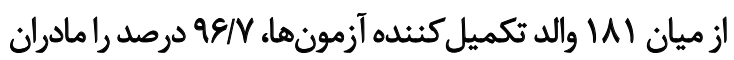

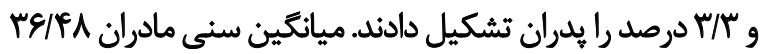

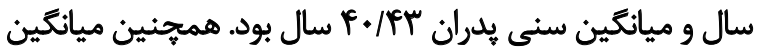

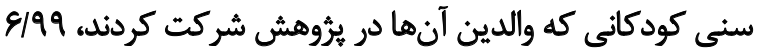

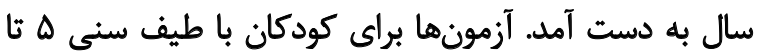
Ir سال اجرا شد كه يس از تعيين فراوانى جنسيث كودكان

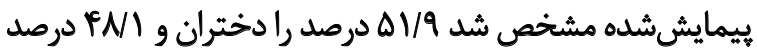

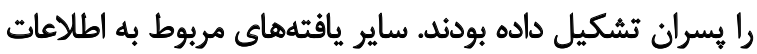

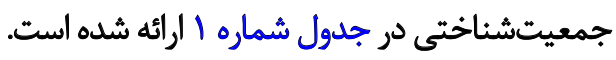

جلول شماره Y شامل مشخصهواي آمارى ميانئين و انحر افمعيار ونيز ضرايب همبستكى نمرات كل IES-R و زيرمقياس هاى آلى آن است
حاضر هايايى فرم والد CSI-4 از طريق اجراى مجدد آزمون با

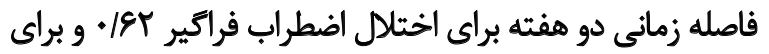

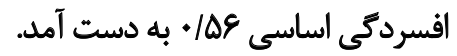

مقياس ثجديدنظر شده ثأثير وويداد (IES-R) اين مقياس يك ابزار ب Yمادهاي است كه در سال 199Y 19 توسط

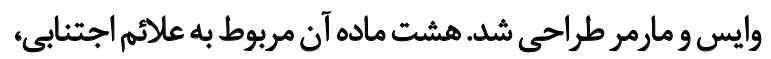

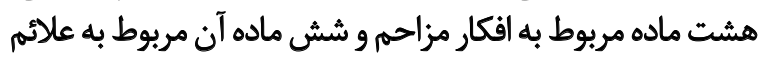

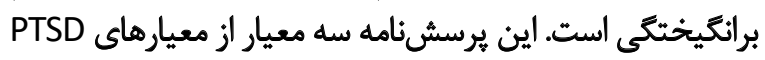

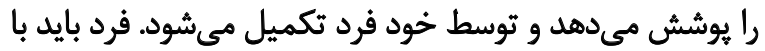

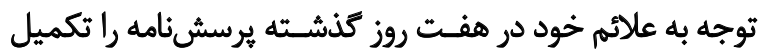

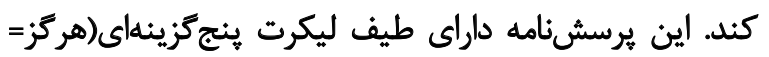

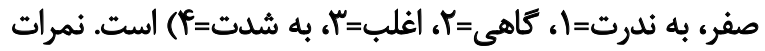

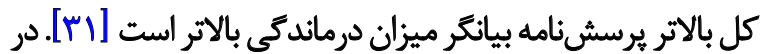

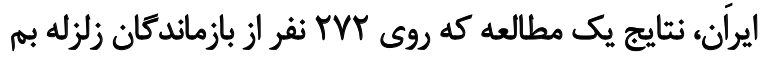

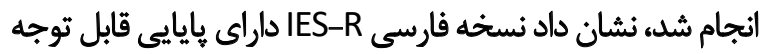

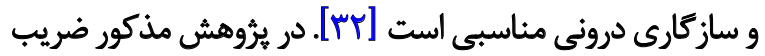

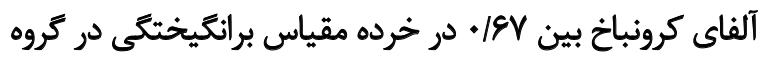

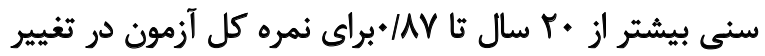

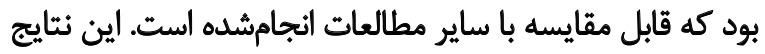
نشاندهنده يايايى نسخه فارسى اين مقياس است. همجنين 18. Impact of Event Scale - Revised (IES-R) 
جدول F. ركرسيونهاى خطى سلسلهمراتبى براى بيشبينى نمرات اضطراب و افسردئى بر اساس نمرهكل IES-R بـ از كنترل عوامل مخدوش كننده جمعيتشناختى

\begin{tabular}{|c|c|c|c|c|c|c|c|}
\hline DW $W^{b}$ & $\mathbf{P}$ & Beta to Enter & F Change & $\mathbf{R}^{2}$ Change & $\mathbf{R}^{2}$ & كامها و متغيرها & متغير ملاك \\
\hline \multirow[t]{2}{*}{$1 / 9.1$} & $.1 . \pi r$ & - & F/VAV &.$/ . P Y$ & .1 .09 & الجعاد فميتشناختى & \multirow[t]{2}{*}{ اضطراب } \\
\hline & .1 .11 &.$/ T A A$ & SINEA & .1 .19 & ג & IES-R نمرهكل & \\
\hline \multirow[t]{2}{*}{ 1/arf } & $.1 .4 A$ & ./IFY & r/q\&8 & \% $/ 4 r$ & r & الجمعيتشناختى & \multirow[t]{2}{*}{ افسردكى } \\
\hline & $.1 \ldots 1$ & . & $r / / 2 q T$ & $. / 1 . \Delta$ & t/Ir & r. نمرهكل IES-R & \\
\hline
\end{tabular}

كنترل متغيرهاى جمعيتى نشان مى دهد.

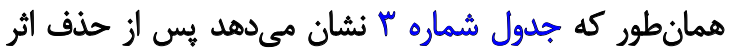

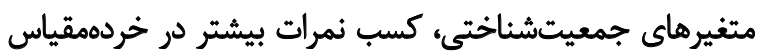

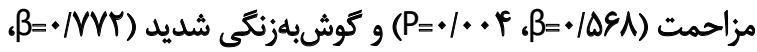

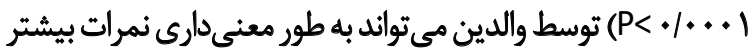

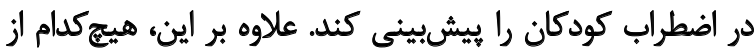

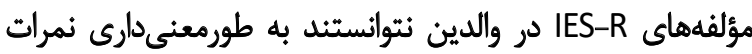

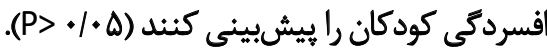
در تحليلهاى ركرسيون جندمثغيرى مجزا، ديكر نمرات

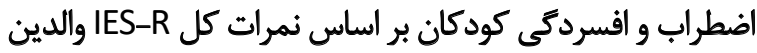

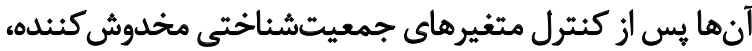

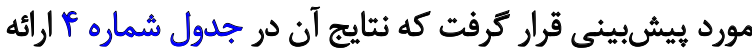

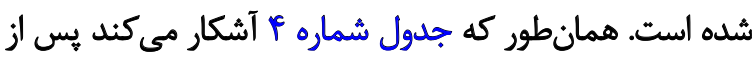

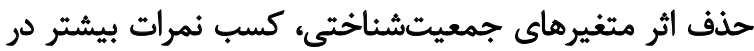

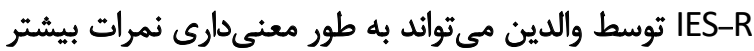

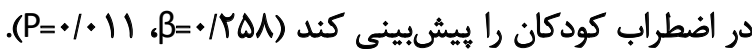

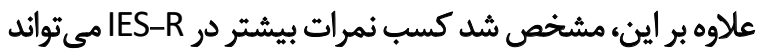

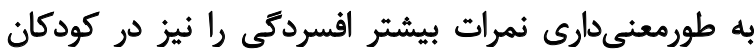

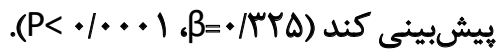

بحث

يافتهماى يُوهش حاضر نشان دادند بين افكار مزاحم و

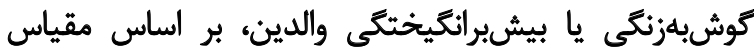

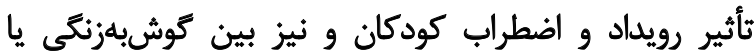

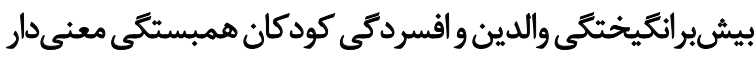

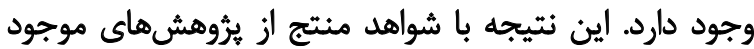

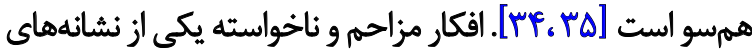

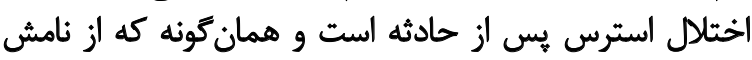

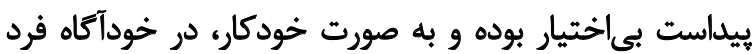

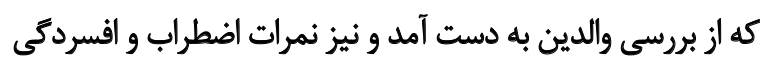

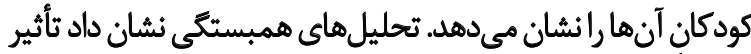

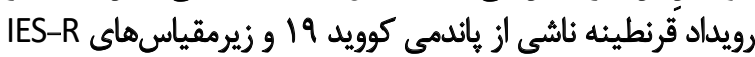

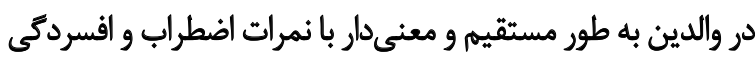

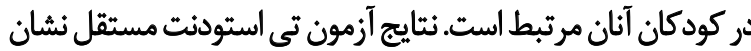

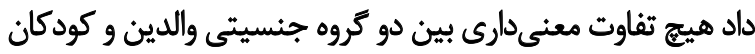

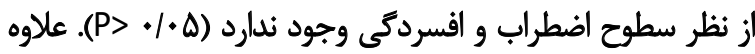

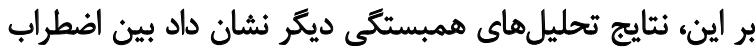

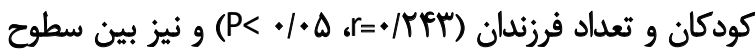

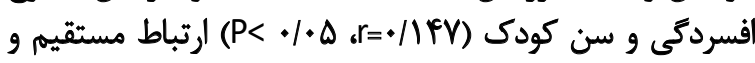

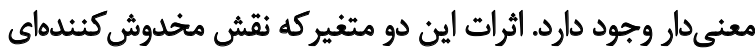

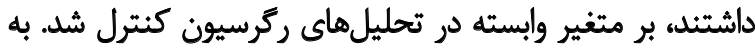

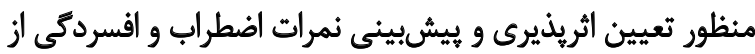

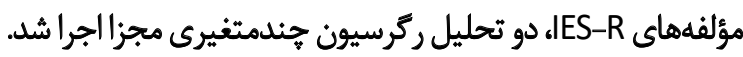

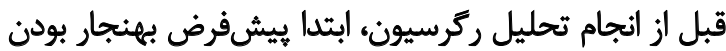

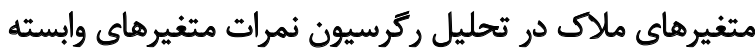

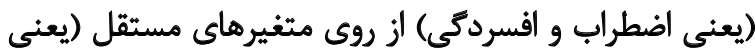

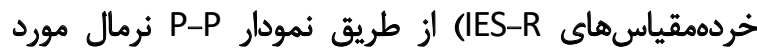

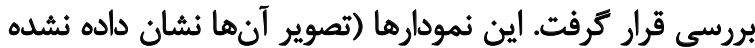

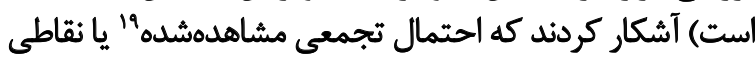

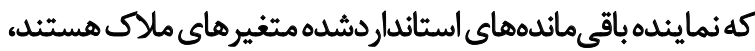

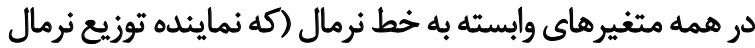

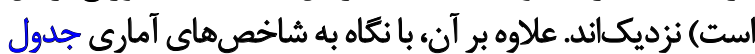

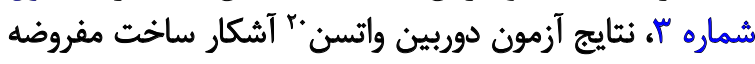

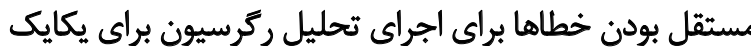

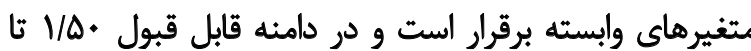

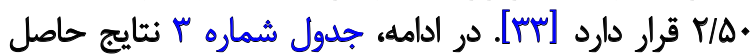

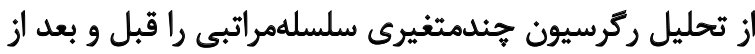

19. Observed cumulative probability 20. Durbin-Watson test (DW) 


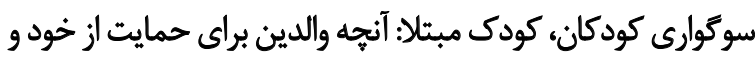
خانواده انجام دهند و غيره) و اقدامات كودينا درازمدان

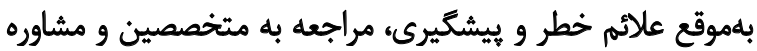

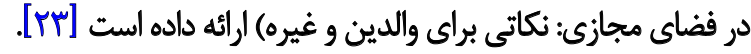

\section{5 sorit}

با توجه به نتايج اين تحقيق و تأييد ثأثير افكار ناخواسته و

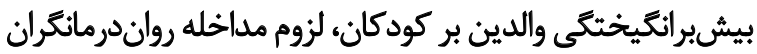

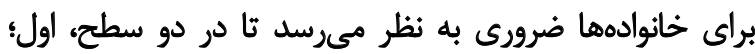

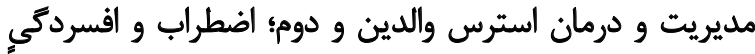

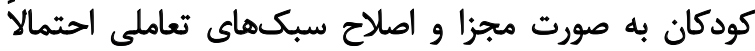

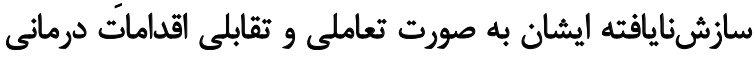

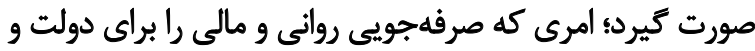
متوليان بهداشت و درمان به دنبال دارد.

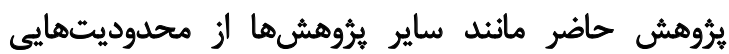

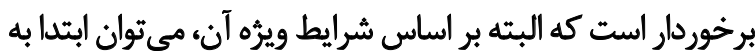

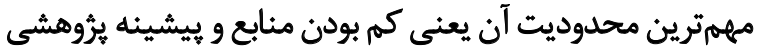

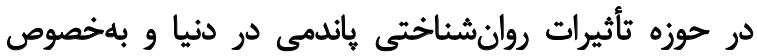

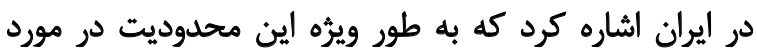

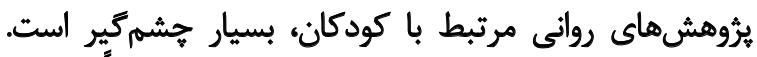

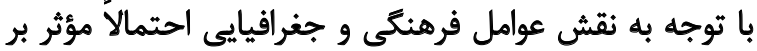

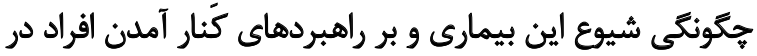

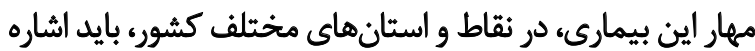

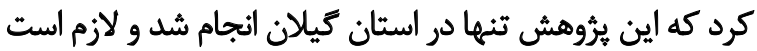

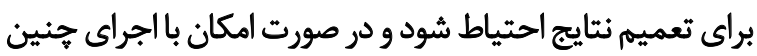

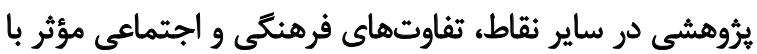

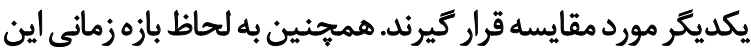

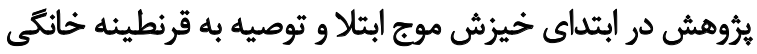

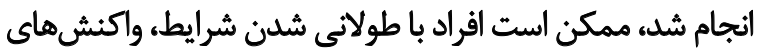

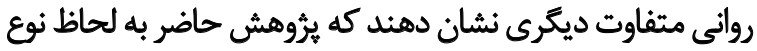

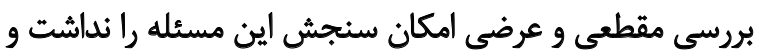

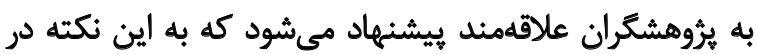

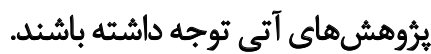

از ديكر محدوديتهاى يرُوهش ميتوان به به شيوه اجراي

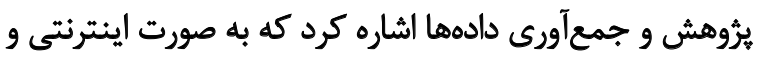

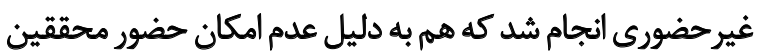

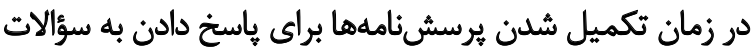

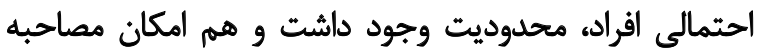

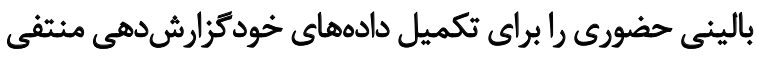

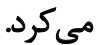

ظاهر مي شود. خاطرات مزاحم و استرس آور مى توانند با بازآرايى

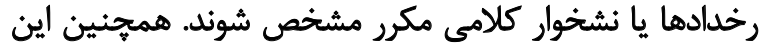

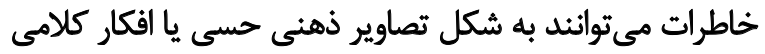

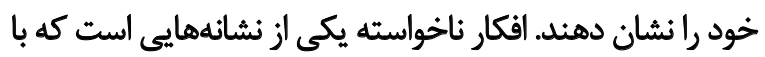

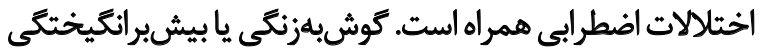

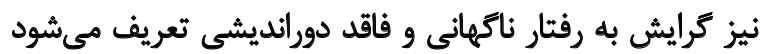

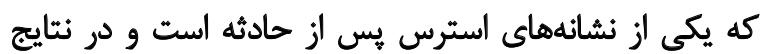

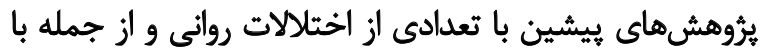
اضطراب و افسردىى همبردى دارد [عبـ] به نظر مىرسد ترس از بيمارى، مشكلات مالى ناشى از قرنطينه

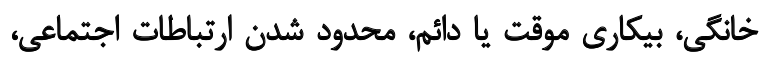

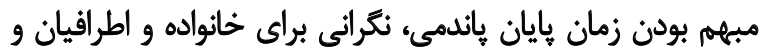

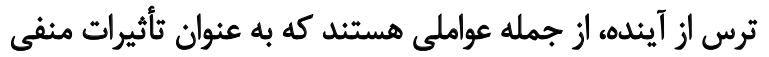

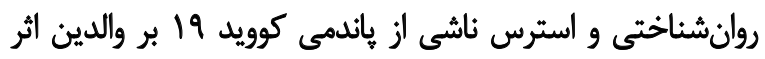

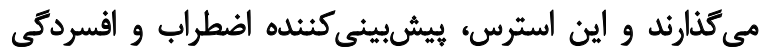

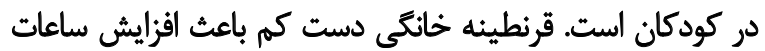

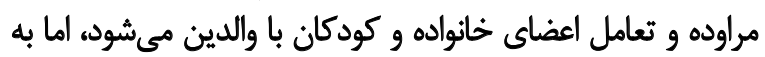

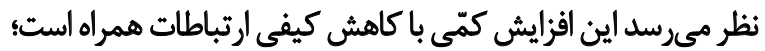

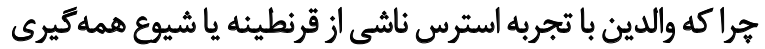

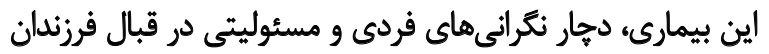

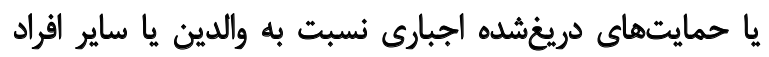

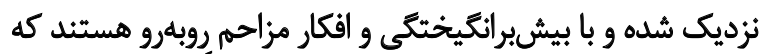

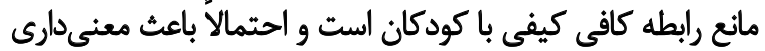

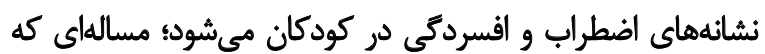
نياز به مطالعات بيشتر آتى با طرحهاي نيمها آزمايشى داردي دارد. با توجه

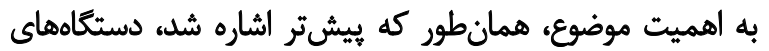

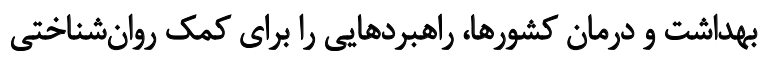

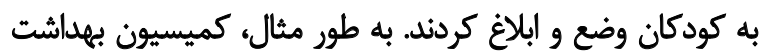

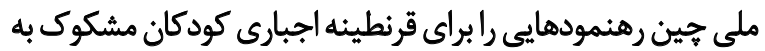

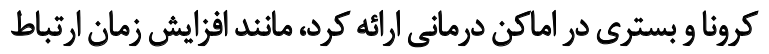

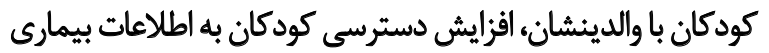

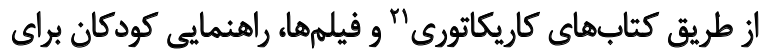

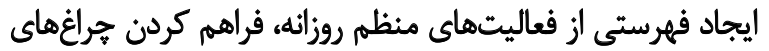

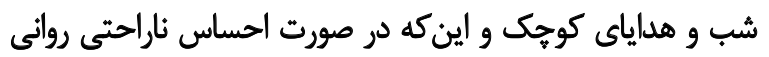

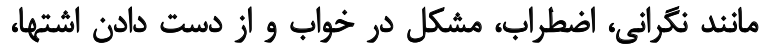

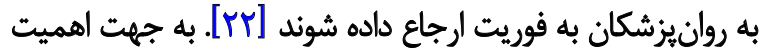

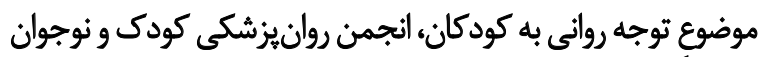

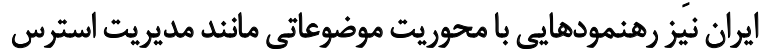

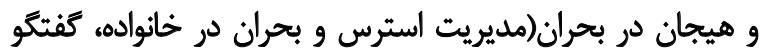

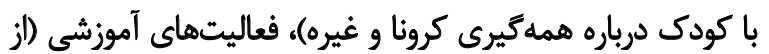

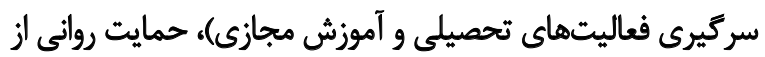

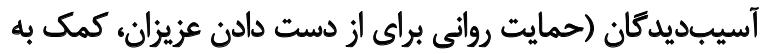




\section{ماحظات اخالاقى}

$$
\text { بيروى از اصول اخلاق يُوهش }
$$

در اين مطالعه، افراد به طور آكاهانه و با رضايت شخصى وائ وارد

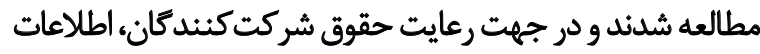

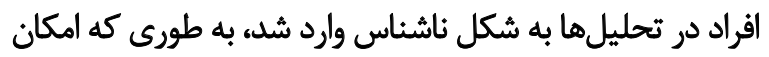

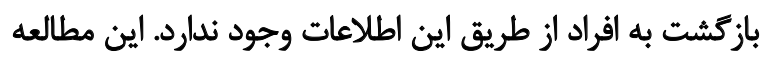

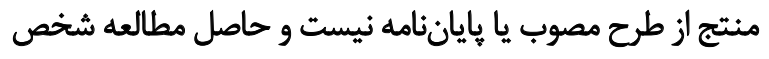

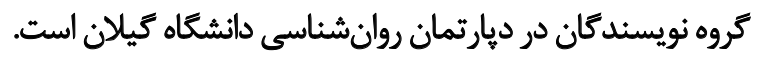

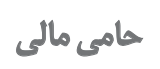

اين برؤوه هيج كونه كمك مالى از سازمانهاى دولتى،

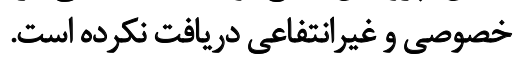

$$
\text { مشار كث نويسندكَان }
$$

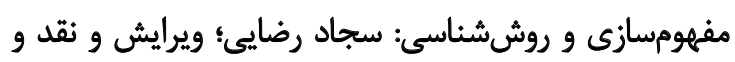

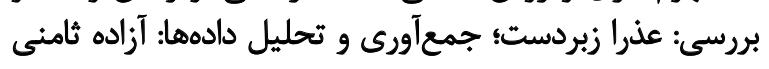

$$
\text { توسروندانى. }
$$

بنابر اظهار نظر نويسندكان، اين مقاله تعارض منافع ندارد. 


\section{References}

[1] World Health Organization. Q\&A on Coronaviruses(COVID-19). [Internet]. 2020 [Updated 2020 April 17]. https:/ / www.who.int/ news-room/q-a-detail/q-a-coronaviruses. page

[2] Ministry of Health and Medical Education(MOHME). [Internet]. 2020 [Updated 2020 February 2]. http://ird.behdasht.gov.ir/ page/News/pagenumber/129. Page

[3] Brooks SK, Webster RK, Smith LE, Woodland L, Wessely S, Greenberg N, et al. The psychological impact of quarantine and how to reduce it: Rapid review of the evidence. Lancet. 2020; 395:912-20. [DOI:10.1016/ S0140-6736(20)30460-8.]

[4] Arefi MF, Poursadeqiyan M. A review of studies on the COVID-19 epidemic crisis disease with a preventive approach. Work. 2020; 66(4):717-29. [DOI:10.3233/WOR-203218] [PMID]

[5] Xiang Y-T, Yang Y, Li W, Zhang L, Zhang Q, Cheung T, Ng $\mathrm{CH}$. Timely mental health care for the 2019 novel coronavirus outbreak is urgently needed. Lancet Psychiatry. 2020; 7(3):228-9. [DOI:10.1016/S2215-0366(20)30046-8]

[6] Huang Y, Zhao N. Generalized anxiety disorder, depressive symptoms and sleep quality during COVID-19 epidemic in China: A web-based cross- sectional survey. Psychiatry Research. 2020; 288:112954. [DOI: 10.1016/j.psychres.2020.112954]

[7] Wang Y, Xu B, Zhao G, Coa R, He X, Fu S. Is quarantine related to immediate negative psychological consequences during the 2009 H1N1 epidemic? General Hospital Psychiatry. 2011; 33(1):75-7. [DOI:10.1016/j.genhosppsych.2010.11.001] [PMID]

[8] Liu X, Kakade M, Fuller CJ, Fan B, Fang Y, Kong J, et al. Depression after exposure to stressful events: Lessons learned from the serve acute respiratory syndrome epidemic. Comprehensive Psychiatry. 2012; 53(1):15-23. [DOI:10.1016/j.comppsych.2011.02.003] [PMID] [PMCID]

[9] Hossain MM, Sultana A, Purohit N. Mental health outcomes of quarantine and isolation for infection prevention: A systematic umbrella review of the global evidence. Epidemiol Health. 2020; 42:e2020038. [DOI: https://doi.org/10.4178/epih.e2020038 ]

[10] LeeSM, Kang WS, Cho AR, Kim T, Park JK. Psychological impact of the 2015 MERS outbreak on hospital workers and quarantined hemodialysis patients. Comprehensive Psychiatry. 2018; 87:123-7. [DOI:10.1016/j.comppsych.2018.10.003] [PMID] [PMCID]

[11] Cenat JM, Mukunzi JN, Noorishad P, Rousseau C. A systemic review of mental health program population affected by the Ebola virus disease. Journal of Psychosomatic Research. 2020; 131:109966. [DOI:10.1016/j.jpsychores.2020.109966] [PMID]

[12] Gao J, Zheng P, Jia Y, Chen H, Mao Y, Chen S, et al. Mental health problems and social media exposure during COVID-19 outbreak. Plos One. 2020; 15(4):e0231924. [DOI: 10.1371/journal. pone.0231924]

[13] American Psychiatric Association. Diagnostic and Statistical Manual of Mental Disorders. Washington, DC.: American Psychiatric Association; 2013. [DOI:10.1176/appi.books.9780890425596]

[14] Qeshta H, Hawajri AM, Thabet AM. The relationship between war trauma, PTSD, anxiety and depression among adolescents in the Gaza Strip. Health Science Journal. 2019; 13(1):621. [DOI:10.21767/1791-809X.1000621]
[15] Mason JE, LeBouthillier DM, Asmundson GJG. Relationships between health behaviors, posttraumatic stress disorder, and comorbid general anxiety and depression. Cognitive Behaviour Therapy. 2019; 48(3):184-99. [DOI:10.1080/16506073.2018.149811 9] [PMID]

[16] Sepahvand T. [Depression and social anxiety in primary school children in the context of cognitive flexibility of mothers (Persian)]. Journal of Arak University of Medical Sciences. 2019; 22(2):57-66. http://jams.arakmu.ac.ir/article-1-5891-fa.html.

[17] Moosavi SM, Ahmadi M. [Behavioral disorders in children with major depressive mothers(Persian)]. Journal of Gorgan University of Medical Sciences. 2012; 14(3):115-20. http://goums. ac.ir/journal/article-1-1414-fa.html

[18] Sprang G, Silman M. Posttraumatic stress disorder in parents and youth after health-related disasters. Disaster Medicine and Public Health Preparedness. 2013; 7(1):105-10 [DOI:10.1017/ dmp.2013.22] [PMID]

[19] Wang G, Zhang Y, Zhao J, Zhang J, Jiang F. Mitigate the effects of home confinement on children during the COVID-19 outbreak. The Lancet. 2020; 395(10228):945-7. [DOI:10.1016/S01406736(20)30547-X]

[20] Liu JJ, Bao Y, Huang X, Shi J, Lu L. Mental health considerations for children quarantined because of COVID-19. The Lancet Child \& Adolescent Health. 2020; 4(5):347-9. [DOI:10.1016/ S2352-4642(20)30096-1.]

[21] Norredam M, Nellums L, Nielsen RS, Byberg S, Petersen JH Incidence of psychiatric disorders among accompanied and unaccompanied asylum seeking children in Denmark: A nationwide register-based cohort study. European Child \& Adolescent Psychiatry. 2018; 27(4):439-46. [DOI:10.1007/s00787-018-1122-3] [PMID]

[22] Devex. National Health Commission of the People's Republic of China. 2021 [Updated 2021 February 25]. Available from: https://www.devex.com/organizations/national-health-commission-of-the-people-s-republic-of-china-1320921

[23] Iranian Academy of Child and Adolescent Psychiatry. Intervention in the epidemic crisis of the COVID virus-19. [Internet]. 2020 [Updated 2020 April 2]. Available from: http:/ /iacap.ir/cate gory $/ \% \mathrm{~d} 8 \% \mathrm{a} 7 \% \mathrm{~d} 8 \%$ ae $\% \mathrm{~d} 8 \% \mathrm{a} 8 \% \mathrm{~d} 8 \% \mathrm{a} 7 \% \mathrm{~d} 8 \%$ b1/. Page

[24] Poursharifi H, Babapour J, Aliloo MM, Khanjani Z, Zeinali S. [Designing and testing a child anxiety model based on child and parent variables (Persian)]. Journal of Family Psychology. 2017; 4(1):17-28. http://ijfp.ir/article-1-235-fa.html.

[25] Shojaei Z, Golparvar M, Aghaei A, Bordbar MR. [Comparing the effectiveness of group story therapy and the art-play therapy on anxiety and depression in children with cancer: Based on the framework, principles and rules of cognitive-behavioral approach (Persian)]. Iranian Journal of Rehabilitation Research in Nursing. 2019; 6(1):50-9. http:/ /ijrn.ir/article-1-438-fa.html.

[26] Murray CJS. A collaborative approach to meeting the psychosocial needs of children during an influenza pandemic. Journal for Specialists in Pediatric Nursing. 2010; 15(2):135-43. [DOI:10.1111/ j.1744-6155.2009.00229.x] [PMID]

[27] Saffarinia, M. [The prediction of mental health based on the anxiety and the social cohesion that caused by Coronavirus (Persian)]. Social Psychology Research. 2020; 9(36):129-41. http:// www.socialpsychology.ir/article_105547.html?lang=en 
[28] Faul F, Erdfelder E, Buchner A, Lang AG. Statistical power analyses using $\mathrm{G} \times$ Power 3.1: Tests for correlation and regression analyses. Behavior Research Methods. 2009; 41(4):1149-60. [DOI:10.3758/BRM.41.4.1149] [PMID]

[29] Gadow KD, Sprafkin J. Quick Guide to Using the Youth's Inventory-4 Screening Kit. Stony Brook, NY: Checkmate Plus; 1997.

[30] Mohamadesmaiel E, Alipour A. [A preliminary study on the reliability, validity and cut off points of the disorders of Children Symptom Inventory-4 (CSI-4) (Persian)]. Journal of Exceptional Children. 2002; 2(3):239-54. http:/ /joec.ir/article-1-484-en.html

[31] Weiss DS, Marmar CR. The Impact of Event Scale-Revised. In: Wilson JP, Keane TM, editors. Assessing psychological trauma and PTSD. New York: Guilford Press; 1997. [DOI:10.1037/t12199-000]

[32] Panaghi L, Hakim Shoshtari M, Atari Moghadam J. [Persian version validation in impact of event-revised (Persian)]. Tehran University Medical Journal. 2006; 64(3):52-60. https:/ / tumj.tums. ac.ir $/$ browse.php?a_id=974\&sid=1\&slc_lang=en

[33] Coakes S, Steed L, Ong C. SPSS without anguish. Milton, QLD: Wiley; 2010

[34] Weissman MM, Leckman JF, Merikangas KR, Gammon GD, Prusoff BA. Depression and anxiety disorders in parents and children: Results from the yale family study. Archives of General Psychiatry. 1984; 41(9):845-52. [DOI:10.1001/ archpsyc.1984.01790200027004] [PMID]

[35] Woodruff-Borden J, Morrow C, Bourland S, Cambron S. The behavior of anxious parents: Examining mechanisms of transmission of anxiety from parent to child. Journal of Clinical Child and Adolescent Psychology. 2002; 31(3):364-74. [DOI:10.1207/ S15374424JCCP3103_08] [PMID]

[36] Hosseinpour N, Mizahossein H, Zarghami Hajebi M, Monirpour N. [Investigating the effect of safe attachment stimulation in the level of subconscious in reducing the disturbing memories in students with Generalized Anxiety Disorder (Persian)]. Journal of Psychoscience. 2018; 17(70):729-34. http://psychologicalscience. ir/article-1-283-fa.html. 